\title{
KECK SPECTROSCOPY OF THE GRAVITATIONAL LENS SYSTEM PG 1115+080: REDSHIFTS OF THE LENSING GALAXIES ${ }^{1}$
}

\author{
TOMISLAV KUNDIĆ
}

Theoretical Astrophysics, California Institute of Technology, Mail Code 130-33, Pasadena, California 91125

Electronic mail: tomislav@tapir.caltech.edu

\section{JUDITH G. COHEN}

Palomar Observatory, California Institute of Technology, Mail Code 105-24, Pasadena, California 91125 Electronic mail: jlc@astro.caltech.edu

\section{ROGER D. BLANDFORD}

Theoretical Astrophysics, California Institute of Technology, Mail Code 130-33, Pasadena, California 91125 Electronic mail: rdb@tapir.caltech.edu

LORI M. LUBIN

The Observatories of the Carnegie Institution of Washington, 813 Santa Barbara Street, Pasadena, California 91101 Electronic mail: lml@ociw.edu Received 1997 April 15; revised 1997 May 13

\begin{abstract}
The quadruple system PG $1115+080$ is the second gravitational lens with a reported measurement of the Hubble constant. In addition to the primary lens, three nearby galaxies are believed to contribute significantly to the lensing potential. In this paper we report accurate redshifts for all four galaxies and show that they belong to a single group at $z_{d}=0.311$. This group has very similar properties to Hickson's compact groups of galaxies found at lower redshifts. We briefly discuss implications for the existing lens models and derive $H_{0}=52 \pm 14 \mathrm{~km} \mathrm{~s}^{-1} \mathrm{Mpc}^{-1}$. (C) 1997 American Astronomical Society. [S0004-6256(97)02908-7]
\end{abstract}

\section{INTRODUCTION}

Gravitational lensing provides a method of measuring Hubble's constant at cosmological distances independently of the traditional distance ladder. In a multiply-imaged source the difference in geometrical path length and gravitational potential along each light ray results in a time delay which can be measured if the source is variable. This time delay is inversely proportional to $H_{0}$ with the constant of proportionality that depends on the mass distribution in the lens (Refsdal 1964).

Though simple in principle, Refsdal's method has been difficult to implement, because of demanding observations required to determine the time delays. In the double quasar 0957+561 A,B (Walsh et al. 1979), there has been a long controversy over the correct value of the delay (Haarsma et al. 1997, and references therein) that was only recently resolved by Kundic et al. $(1995,1996)$. Combined with the lens model of Grogin \& Narayan (1996), the galaxy velocity dispersion of Falco et al. (1997), and the cluster mass model of Fischer et al. (1996), this time delay yields $H_{0}=64$ $\pm 13 \mathrm{~km} \mathrm{~s}^{-1} \mathrm{Mpc}^{-1}$.

The second lens with a known time delay is the quadruple

${ }^{1}$ Based on observations obtained at the W. M. Keck Observatory, which is operated jointly by the California Institute of Technology and the University of California. quasar PG 1115+080 (Schechter et al. 1997; Bar-Kana 1997). This system consists of four images of a $z_{s}=1.722$ quasar (Weymann et al. 1980) lensed by a foreground group of galaxies at $z_{d} \sim 0.3$ (Henry \& Heasley 1986; AngoninWillaime et al. 1993). HST imaging of PG $1115+080$ established the relative positions of the four quasar images with an uncertainty of 5 mas and the centroid of the lensing galaxy with an uncertainty of 50 mas (Kristian et al. 1993). A schematic diagram of the system is shown in Fig. 1 of Keeton \& Kochanek (1996, hereafter KK96). We adopt their galaxy designations (based on Young et al. 1981), in which the primary lens is named $\mathrm{G}$, and the nearby group members are named G1, G2; and G3.

The goals of this paper are (1) to provide an accurate redshift for the primary lensing galaxy and thus reduce the uncertainty in the derived value of $H_{0},(2)$ to establish that $\mathrm{G}, \mathrm{G} 1, \mathrm{G} 2$, and G3 belong to the same group of galaxies, and (3) to estimate the velocity dispersion of this group.

\section{DATA ACQUISITION}

Optical spectra of PG $1115+080$ were obtained on two nights, 1997 February 7 and 1997 March 2, with the Low Resolution Imaging Spectrometer (Oke et al. 1995) at the Keck II $10 \mathrm{~m}$ telescope. Table 1 lists the relevant observing parameters. The first two exposures were taken with the 300 line/mm grating and the $0.7^{\prime \prime}$ slit, and the other three with the

\section{(C) American Astronomical Society - Provided by the NASA Astrophysics Data System}


TABLE 1. Observing parameters.

\begin{tabular}{ccccccccc}
\hline \hline $\begin{array}{c}\text { Exposure } \\
\text { Number }\end{array}$ & Object & $\begin{array}{c}\text { UT } \\
\text { Date }\end{array}$ & $\begin{array}{c}\text { UT } \\
\text { Time }\end{array}$ & Airmass & $\begin{array}{c}\text { P.A. } \\
\left({ }^{\circ} \text { E of N) }\right)\end{array}$ & $\begin{array}{c}\text { Exposure } \\
\text { Time (s) }\end{array}$ & Grating & $\begin{array}{c}\text { Wavelength } \\
\text { Range }(\AA)\end{array}$ \\
\hline 1 & G & 1997 Feb 7 & $09: 51$ & 1.31 & 298 & 600 & $300 / 5000$ & $3900-8800$ \\
2 & G & 1997 Feb 7 & $10: 04$ & 1.26 & 298 & 600 & $300 / 5000$ & $3900-8800$ \\
3 & G2, G3 & 1997 Mar 2 & $09: 02$ & 1.16 & 192 & 1200 & $600 / 5000$ & $4800-7300$ \\
4 & G2, G3 & 1997 Mar 2 & $09: 31$ & 1.10 & 192 & 1200 & $600 / 5000$ & $4800-7300$ \\
5 & G1, G3 & 1997 Mar 2 & $09: 54$ & 1.06 & 280 & 1200 & $600 / 5000$ & $4800-7300$ \\
\hline \hline
\end{tabular}

600 line/mm grating and the $1.0^{\prime \prime}$ slit. The resulting spectral resolution, as measured from unresolved sky lines, was approximately $7 \AA$ in the low-resolution spectra and $4.5 \AA$ in the high-resolution spectra. The seeing was $\sim 0.7^{\prime \prime}$ in exposures 1 and 2 , and $\sim 1.2^{\prime \prime}$ in exposures $3-5$.

The spectra were reduced in a standard fashion using the "longslit" and "apextract" packages in IRAF. ${ }^{2}$ In the case of the main lensing galaxy $G$, we had to subtract the contaminating quasar light from the galaxy spectrum. Galaxy $G$ is located approximately halfway between images $\mathrm{A}$ and $\mathrm{C}$, which are separated by about 2 arcsec. In the $R$ band, where the spectrograph is most sensitive, image $\mathrm{A}^{3}$ is $\sim 4 \mathrm{mag}$ brighter than $\mathrm{G}$, and image $\mathrm{C}$ is $\sim 2$ mag brighter. We thus proceeded by first extracting the image $A$ spectrum and then shifting the trace by 1 and 2 arcsec to extract the spectra of galaxy $\mathrm{G}$ and image $\mathrm{C}$. The contribution of image $\mathrm{A}$ to the galaxy aperture was then subtracted using an aperture on the opposite side of the image A center. The same was repeated for image $\mathrm{C}$. The resulting galaxy spectrum is shown in the bottom panel of Fig. 1, while the spectra of quasar images A and $\mathrm{C}$ are shown in the top two panels. Note that no correction for galaxy contamination was made to the quasar fluxes. Some of the stronger galaxy absorption lines can thus be seen in the image $C$ spectrum.

For each spectrum the wavelength solution was derived using sky emission lines identified in the atlas of Osterbrock et al. (1996). After fitting a 4th-order Legendre polynomial to 10-20 strong, isolated lines, the rms residuals were typically $\leqq 0.3 \AA$. In order to minimize systematic errors, the sky spectra were extracted on both sides of each galaxy spectrum, independently calibrated and averaged for the final wavelength solution. The shift between the two calibrations was in all cases smaller than $0.5 \AA$.

Approximate redshifts of the lensing galaxies were first estimated from strong absorption features in the spectra, particularly the $\mathrm{Ca}$ II $\mathrm{H}$ and $\mathrm{K}$ lines and the $\mathrm{G}$ band. These features, along with the Balmer series lines and the MG Ib triplet, are marked with dotted lines in Fig. 2. Accurate redshifts were then determined by cross-correlating the galaxy spectra with stellar templates of Jones (1996) available on the AAS CD-ROM Series, Vol. 7 (Leitherer et al. 1997). Only $\mathrm{G}$ and $\mathrm{K}$ giants were used, since they provide the closest match to the spectral characteristics of the early type

${ }^{2}$ IRAF is distributed by the National Optical Astronomy Observatories, which are operated by the Association of Universities for Research in Astronomy, Inc., under cooperative agreement with the National Science Foundation.

${ }^{3}$ Image A consists of a close pair of images A1 and A2 which were unresolved in the original discovery paper (Weymann et al. 1980). galaxies in the lensing group. The results of the crosscorrelation analysis are summarized in Table 2. The errors listed in the table were calculated from the width of the cross-correlation peak (Tonry \& Davis 1979) and an estimate of the systematic error in wavelength calibration. Our results are consistent with the previous measurement of the G1 and G2 redshifts by Henry \& Heasley (1986), and marginally consistent (at $3 \sigma$ ) with the $\mathrm{G}$ redshift reported by AngoninWillaime et al. (1993).

\section{PROPERTIES OF THE LENSING GROUP}

The spectra of G, G1, G2, and G3 clearly show that they belong to a single group of galaxies at the redshift of $z_{d}$ $=0.311$ (Fig. 2). The rest-frame line-of-sight velocity dispersion of this group is $\sigma_{v}=c \sigma_{z} /(1+z)=270 \pm 70 \mathrm{~km} \mathrm{~s}^{-1}$, where the formal error includes only the uncertainty in the individual galaxy redshifts. Because of the small number of

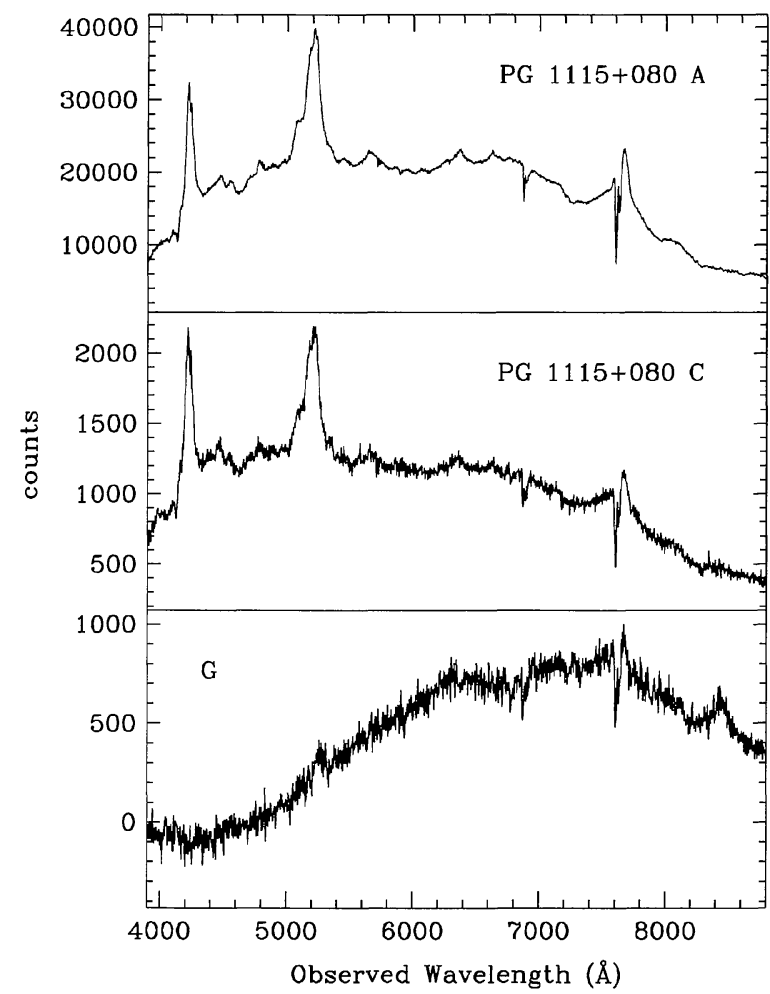

FIG. 1. The spectra of PG $1115+080$ images A (top) and C (middle), and the primary lensing galaxy $\mathrm{G}$ (bottom). Broad emission line residuals in the $G$ spectrum are caused by imperfect quasar subtraction. The spectra have not been flux-calibrated. 


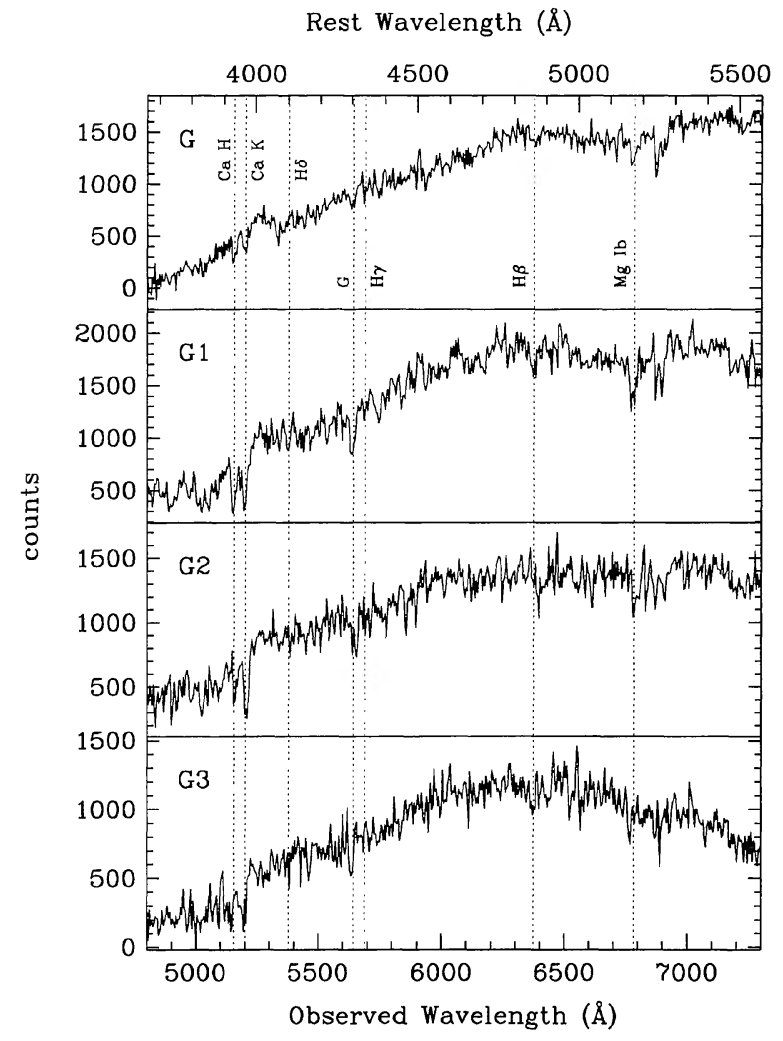

FIG. 2. The spectra of the primary lens $\mathrm{G}$ and group galaxies G1, G2, and G3 (top to bottom) binned to $5 \AA$ resolution. Strong spectral features are identified with dotted lines assuming the group redshift of $z_{d}=0.311$. The same redshift is used for the rest wavelength scale on the top of the figure. The redshift difference between $\mathrm{G} 2$ and the other three galaxies is noticeable.

galaxies used to derive $\sigma_{v}$, the velocity dispersion of the mass associated with the lensing group could be substantially larger (Ramella et al. 1994).

Properties of the lensing group in PG $1115+080$ are very similar to those of Hickson's compact groups (HCGs) of galaxies (Hickson 1982; Hickson et al. 1992). The median projected separation of galaxies in the lensing group is

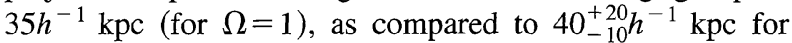
HCGs (we quote the median with upper and lower quartiles). The one-dimensional velocity dispersion of $270 \mathrm{~km} \mathrm{~s}^{-1}$ is also within the range of $200_{-80}^{+100} \mathrm{~km} \mathrm{~s}^{-1}$ characteristic of HCGs. The absolute magnitudes of the lensing galaxies are less certain, but if we adopt $M_{B}=-19.1$ for the primary lens (KK96), the range of magnitudes in the lensing group, $-20 \lesssim M_{B} \lesssim-19$, is compatible with $M_{B}(\mathrm{HCG})$ $=-19.5_{-0.7}^{+0.8}$.

The gravitational potential associated with the group is crucial for the models of PG $1115+080$, because it provides an independent source of shear required to obtain a statistically acceptable fit to the observables (KK96; Keeton et al. 1996). As in the case of Q $0957+561$, however, the presence of an extended perturber introduces a degeneracy into the models (Falco et al. 1985) that can only be removed if the mass of the primary lens or the perturbing group are independently measured. These estimates can be obtained
TABLE 2. Lensing galaxy redshifts.

\begin{tabular}{ccccc}
\hline \hline Galaxy & $-\Delta \alpha\left(^{\prime \prime}\right)$ & $\Delta \delta\left(^{\prime \prime}\right)$ & Magnitude & Redshift \\
\hline G & -0.4 & -1.3 & $R=20.2$ & $0.3100 \pm 0.0005$ \\
G1 & 20.1 & -12.3 & $r=19.0$ & $0.3099 \pm 0.0005$ \\
G2 & 11.5 & -2.1 & $r=20.0$ & $0.3120 \pm 0.0005$ \\
G3 & 13.8 & -13.5 & $r=20.5$ & $0.3093 \pm 0.0005$ \\
\hline \hline
\end{tabular}

Galaxy positions are given relative to image $C$, based on the data from Young et al. (1991) and Kristian et al. (1993). The magnitude of G is taken from Christian et al. (1987), while the other three magnitudes are adopted from Young et al. (1991).

from the line-of-sight velocity dispersions of the galaxy and the group. The former requires high signal-to-noise, moderate $(\sim 1 \AA)$ resolution spectroscopy of the lensing galaxy (e.g., Falco et al. 1997), a challenging observation because of the proximity of bright quasar images. The velocity dispersion of the group can be improved from our current estimate if additional galaxies associated with the group are found in the vicinity of the lens (Ramella et al. 1994). A deeper spectroscopic survey of the field would thus be highly desirable.

\section{IMPLICATIONS FOR THE HUBBLE CONSTANT}

In the expression for the gravitational lens time delay, it is common to separate the lens model dependence from the cosmological scale factor (e.g., Blandford \& Narayan 1992):

$$
\tau(\boldsymbol{\theta})=\frac{\left(1+z_{d}\right)}{c} \frac{D_{d} D_{s}}{D_{d s}}\left[\frac{(\boldsymbol{\theta}-\boldsymbol{\beta})^{2}}{2}-\Psi(\boldsymbol{\theta})\right],
$$

where $\boldsymbol{\theta}$ and $\boldsymbol{\beta}$ are the image and source positions; $\Psi(\boldsymbol{\theta})$ is the scaled surface potential; and $D_{d}, D_{s}$, and $D_{d s}$ are the angular diameter distances observer-lens, observer-source, and lens-source. In a given lens model specified by $\Psi(\boldsymbol{\theta})$, the value of the Hubble constant derived from the differential time delays, $\tau\left(\boldsymbol{\theta}_{i}\right)-\tau\left(\boldsymbol{\theta}_{j}\right)$, is inversely proportional to $K$ $=\left(1+z_{d}\right) / c\left(D_{d} D_{s}\right) / D_{d s}$. This factor $K$ also depends on the source and lens redshifts, and the choice of cosmological parameters $\Omega_{0}$ and $\Lambda$.

For the source redshift of $z_{s}=1.722$ and the lens redshift of $z_{d}=0.311$, the cosmological scale factor $K$ takes the values of $31.3,33.7,32.6$, and $32.7 h^{-1}$ days $\operatorname{arcsec}^{-2}$ when $\left(\Omega_{0}, \Lambda\right)=(1,0),(0.1,0),(0.4,0.6)$, and $(0.2,0.8)$, respectively. In the $\left(\Omega_{0}, \Lambda\right)=(1,0)$ cosmology, the lens model of KK96 then implies $H_{0}=52 \pm 14 \mathrm{~km} \mathrm{~s}^{-1} \mathrm{Mpc}^{-1}$. This value is approximately 3\% higher than the one quoted by KK96 who use $z_{d}=0.304$. It is worth noting that $K$ is much less sensitive to $\Omega_{0}$ and $\Lambda$ than the angular diameter distances involved, making the derived value of $H_{0}$ robust with respect to the choice of the world model (Blandford \& Kochanek 1987; Blandford \& Kundic 1997). For the same reason, the time delay method is not an effective way to constrain $\Omega_{0}$ and $\Lambda$.

\section{CONCLUSIONS}

In this paper we demonstrate that the main lensing galaxy in the gravitational lens system PG $1115+080$ and its three neighbors belong to a single group at $z=0.311$. With its 
velocity dispersion of $270 \pm 70 \mathrm{~km} \mathrm{~s}^{-1}$ and median projected galaxy separation of $35 h^{-1} \mathrm{kpc}$, this group is very similar to Hickson's compact groups of galaxies discovered at lower redshifts. The presence of the group is important for the models of the system, because it provides an additional source of shear required to explain the observed image configuration. Such a two-shear model has been constructed by
KK96. Using Bar-Kana's (1997) analysis of Schechter et al. (1997) light curves, the KK96 model implies $H_{0}=52$ $\pm 14 \mathrm{~km} \mathrm{~s}^{-1} \mathrm{Mpc}^{-1}$ in an Einstein-DeSitter universe.

We thank D. W. Hogg and S. Malhotra for helpful conversations. This work was supported by NSF Grant No. AST-95-29170 and NASA Grant No. NAG-5-3834.

\section{REFERENCES}

Angonin-Willaime, M.-C., Hammer, F., \& Rigaut F. 1993, in Gravitational Lenses in the Universe, edited by J. Surdej, D. Fraipont-Caro, E. Gosset, S. Refsdal, and M. Remy (Université de Liège, Institut d'Astrophysique Liège, Belgium), p. 85

Bar-Kana, R. 1997, preprint, astro-ph/9701068

Blandford, R. D., \& Kochanek, C. S. 1987, in The Dark Matter in the Universe, edited by J. Bahcall, T. Piran, and S. Weinberg (World Scientific, Singapore), p. 133

Blandford, R. D., \& Kundić, T. 1997, in The Extragalactic Distance Scale, edited by M. Livio, M. Donahue, and N. Panagia (Cambridge University Press, Cambridge) (in press)

Blandford, R. D., \& Narayan, R. 1992, ARA\&A, 30, 311

Christian, C. A., Crabtree, D., \& Waddell, P. 1987, ApJ, 312, 45

Falco, E. E., Gorenstein, M. V., \& Shapiro, I. I. 1985, ApJ, 289, L1

Falco, E. E., Shapiro, I. I., Moustakas, L. A., \& Davis, M. 1997, preprint, astro-ph/9702152

Fischer, P., Bernstein, G., Rhee, G., \& Tyson, J. A. 1996, preprint, astro$\mathrm{ph} / 9608117$

Grogin, N. A., \& Narayan, R. 1996, ApJ, 464, 92 \& 473, 570

Haarsma, D. B., Hewitt, J. N., Lehar, J., \& Burke, B. F. 1997, ApJ, 479, 102

Henry, J. P., \& Heasley, J. N. 1986, Nature, 321, 139

Hickson, P. 1982, ApJ, 255, 382

Hickson, P., Mendes de Oliveira, C., Huchra, J. P., \& Palumbo, G. G. C. 1992, ApJ, 399, 353
Jones, L. 1996, Ph.D. thesis, University of North Carolina at Chapel Hill Keeton, C. R., \& Kochanek, C. S. 1996, preprint, astro-ph/9611216 (KK96) Keeton, C. R., Kochanek, C. S., \& Seljak, U. 1996, preprint, astro-ph/ 9610163

Kristian, J., et al. 1993, AJ, 106, 1330

Kundić, T., Colley, W. N., Gott, J. R. III, Malhotra, S., Pen, U., Rhoads, J. E., Stanek, K. Z., \& Turner, E. L. 1995, ApJ, 455, L5

Kundić, T., et al. 1996, preprint, astro-ph/9610162

Leitherer, C., et al. 1997, PASP, 108, 996

Oke, J. B., et al. 1995, PASP, 107, 375

Osterbrock, D. E., Fulbright, J. P., Martel, A. R., Keane, M. J., \& Trager, S. C. 1996, PASP, 108, 277

Ramella, M., Diaferio, A., Geller, M. J., \& Huchra, J. P. 1994, AJ, 107, 1623

Refsdal, S. 1964, MNRAS, 128, 307

Schechter, P. L., et al. 1997, ApJ, 475, L85

Tonry, J., \& Davis, M. 1979, AJ, 84, 1511

Walsh, D., Carswell, R. F., \& Weymann, R. J. 1979, Nature, 279, 381

Weymann, R. J., Latham, D., Angel, J. R. P., Green, R. F., Liebert, J. W., Turnshek, D. A., Turnshek, D. E., \& Tyson, J. A. 1980, Nature, 285, 641 Young, P., Deverill, R. S., Gunn, J. E., \& Westphal, J. A. 1981, ApJ, 244, 723 